\title{
PHTHALIC ACID: A GREEN, BIODEGRADABLE AND ENVIRONMENTALLY BENIGN NATURE DI- FUNCTIONAL BRØNSTED ACID CATALYST FOR THE ONE-POT SYNTHESIS OF 3, 4-DIHYDROPYRIMIDIN-2- (1H)-ONE DERIVATIVES AND SUBSTITUTED DIHYDRO-2-OXYPYRROLES
}

\author{
FARZANEH MOHAMADPOUR, ${ }^{a}$ MOJTABA LASHKARI, ${ }^{b}$ \\ MALEK TAHER MAGHSOODLOU, ${ }^{a,{ }^{*}}$ REZA HEYDARI, ${ }^{a}$ \\ ${ }^{a}$ Department of Chemistry, Faculty of Science, University of Sistan and Baluchestan, P. O. Box 98135-674 Zahedan, Iran \\ ${ }^{b}$ Faculty of Science, Velayat University, Iranshahr, Iran
}

\begin{abstract}
Phthalic acid as a green, biodegradable economical and environmentally benign nature catalyst for the one-pot three-component Biginelli synthesis of 3,4-dihydropyrimidin-2-(1H)-one derivatives via $\beta$-keto esters, aldehyde derivatives and urea/thiourea under thermal and solvent-free conditions and one-pot four-component domino condensation of substituted dihydro-2-oxypyrrole by reaction of dialkyl acetylenedicarboxylate, formaldehyde and amines (aromatic and aliphatic) under ambient temperature with short reaction times and excellent yields is reported. The most benefits this procedure are such as green, biodegradable, inexpensive and non-toxic catalyst, eco-friendly, high catalytic activity, efficient, easily separation with no column chromatographic separation, simple operational procedures, one-pot, excellent yields, environmentally benign nature.
\end{abstract}

Keywords: Phthalic acid, 3,4-Dihydropyrimidinone, Substituted dihydro-2-oxypyrrole, Biodegradable green catalyst, Multi-component reactions, Green chemistry.

\section{INTRODUCTION}

In recent years, green chemistry, has become to one of the best approach for green and efficient preparation of organic compounds. The special benefits of green chemistry for the synthesis of heterocyclic compounds are using nontoxic substrate and environmentally benign nature. Herein, our recent studies focused on developing of using green catalyst [1-4] in the multi-component reactions.

In the past decades, the most of research have focused on a study for the synthesis of heterocyclic compounds. Organic compounds containing nitrogenheterocyclic rings are important compounds in medicinal chemistry. Recently, because of their specially pharmaceutical and biological activities, compounds with pyrrole rings such as dihydro-2-oxypyrroles and Biginelli [5] reactions for the synthesis of 3,4-dihydropyrimidin-2-(1H)-ones derivatives have attracted much interest in the organic researches for example these compounds have been used as anticancer [6], calcium channel blockers, $\alpha$-1a-antagonists [7], human cytomegalovirus ( HCMV) protease [8], they has been used as Pl-091 [9], many of number alkaloids with biological activities have pyrrole rings [10], And these rings have been used as UCS1025A [11]. In the recent years, several protocols for the preparation of these compounds that is including bronsted or lewis acid catalysts have been reported for example $\mathrm{Cu}(\mathrm{OAc})_{2}$ [12], [Btto][p-TSA] [13], bakers' yeast [14], Calcium Fluoride [15], copper(II) tetrafluoroborate [16], hydrotalcite [17], TBAB [18], hexaaquaaluminium(III) tetrafluoroborate [19], copper(II)sulfamate [20], I $[21], \mathrm{AcOH}[22],\left[\mathrm{n}_{2}-\mathrm{Bu}_{4} \mathrm{~N}\right]$ [HSO $]$ [23], $\mathrm{Al}\left(\mathrm{H}_{2} \mathrm{PO}_{4}\right)_{3}$ [24], oxalic acid [25], $\mathrm{ZrCl}_{4}$ [26], $\mathrm{InCl}_{3}$ [27]. Some of limitation these methodologies are low yields, toxic catalyst, long time reactions, harsh reaction conditions, expensive materials.
Phthalic acid is a biodegradable green [28-29] and environmentally benign nature di-functional chemical compound that the bacteria Pseudomonas sp. P1 degrades phthalic acid [30]. Because of specially pharmaceutical and biological activities these compounds, the development of efficient and simple methodology for the synthesis of 3, 4-dihydropyrimidin-2-(1H)-ones derivatives and substituted dihydro-2-oxypyrroles has become a main goal of our studies and finally, herein, we have reported a simple and mild one-pot approach for the synthesis of 3,4-dihydropyrimidin-2-(1H)-one derivatives using phthalic acid as a biodegradable green and efficient catalyst by means of three-component Biginelli reaction between $\beta$-keto esters, aldehyde derivatives and urea/thiourea under thermal and solvent-free conditions and also, onepot synthesis of substituted dihydro-2-oxypyrrole through a four-component reaction between amines (aromatic or aliphatic), dialkyl acetylenedicarboxylate and formaldehyde under ambient temperature in methanol with excellent yields. The notable benefits of phthalic acid as a di-functional Brønsted acid catalyst in organic compounds synthesis is green, environmentally benign nature, biodegradable, mild, high catalytic activity, inexpensive, non-toxic.

\section{RESULTS AND DISCUSSION}

A green, biodegradable, economical and efficient catalyst for an environmental safe, simple and mild methodology to diverse synthesis of 3,4-dihydropyrimidin-2-( $1 H)$-one derivatives by using of one-pot threecomponent condensation Biginelli reaction of aldehydes derivatives $(\mathbf{1}, 1.0$ $\mathrm{mmol})$, urea/ thiourea $(2,1.5 \mathrm{mmol})$ and ethyl/methyl acetoacetate $(\mathbf{3}, 1.0$ $\mathrm{mmol}$ ) with phthalic acid under solvent-free and thermal conditions is studied (Scheme 1).

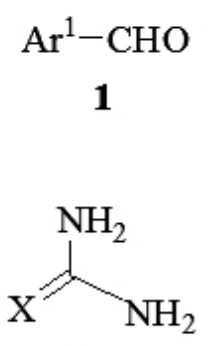

2

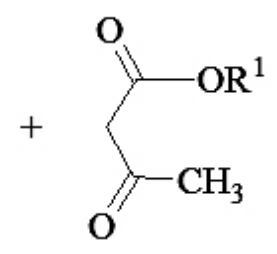

3<smiles>[R]OC(=O)C1=C(C)C([X])=[W]=C(C)NC1[Al]</smiles>

4 a-p

Scheme 1: Synthesis of 3, 4-dihydropyrimidin-2-(1H)-one derivatives 
In order to optimized the reaction conditions, the synthesis of compound $4 \mathbf{a}$ (Table 3 , entry 1 ) was used as a model reaction. The effect of different amount of catalyst on the reaction has been studied in this protocol. No product could be detected in the absence of the catalyst even after $6 \mathrm{~h}$ (Table 1 , entry 1 ). Good yields were obtained in the presence of catalyst. The best amount of catalyst was $15 \mathrm{~mol} \%(0.025 \mathrm{~g})$ (Table1, entry 4$)$. The higher amount of catalyst did not increase the yields products (Table 1, entry 5).

However, the higher yield of product was obtained with $0.025 \mathrm{~g}$ of catalyst and the results are summarized in Table 1.

Table 1: Optimization of the reaction condition $^{a}$

\begin{tabular}{ccccc}
\hline & & & & \\
\hline
\end{tabular}

${ }^{a}$ Reaction conditions: benzaldehyde $(1.0 \mathrm{mmol})$, ethyl acetoacetate $(1.0 \mathrm{mmol})$, urea $(1.5 \mathrm{mmol})$ and phthalic acid was heated at $80^{\circ} \mathrm{C}$ for the appropriate time.

Also, the effect of temperature on the reaction has been studied. No product could be detected in room temperature conditions (Table 2, entry 1 ). The reaction was investigated by changing temperature from $40-100{ }^{\circ} \mathrm{C}$, and the high yield of product is obtained in $80^{\circ} \mathrm{C}$ temperature (Table 2, entry 4 ) and yields of product at different temperature are reported in Table 2.

Table 2: Effect of temperature on the synthesis of $\mathbf{4} \mathbf{a}^{a}$

\begin{tabular}{lcccc}
\hline & & & \\
\hline & & & & \\
\hline
\end{tabular}

${ }^{a}$ Reaction conditions: benzaldehyde $(1.0 \mathrm{mmol})$; ethyl acetoacetate $(1.0 \mathrm{mmol})$; urea $(1.5 \mathrm{mmol})$ and phthalic acid $(15 \mathrm{~mol} \%)$ was heated under various temperatures for the appropriate time.

In order to study of this procedure, we have synthesized a series of compounds with the type of electron-donating and electron-withdrawing aldehydes derivatives such as $\mathrm{Cl}, \mathrm{Br}, \mathrm{NO}_{2}, \mathrm{OH}, \mathrm{OMe}, \ldots$. substituted benzaldehydes which gave excellent yields and the generality of this three condensation reaction was studied by using of phthalic acid $(15 \mathrm{~mol} \%)$ via the type of aldehydes derivatives $(1.0 \mathrm{mmol})$, urea or thiourea $(1.5 \mathrm{mmol})$ and ethyl/methyl acetoacetate $(1.0 \mathrm{mmol})$, under solvent-free conditions at $80{ }^{\circ} \mathrm{C}$ temperature and the results are shown in Table 3.
After the successful synthesis of 3,4-dihydropyrimidin-2-(1H)-one derivatives we turned our attention toward the synthesis of dihydro-2oxypyrroles derivatives by using of a one-pot four-component domino reaction via amines (aromatic or aliphatic $\mathbf{5}$ and 7, $2.0 \mathrm{mmol}$ ), dialkyl acetylenedicarboxylate $(6,1.0 \mathrm{mmol})$ and formaldehyde $(8,1.5 \mathrm{mmol})$ in the presence of phthalic acid as a biodegradable green catalyst under ambient temperature with excellent yields and short reaction times (Scheme 2). 
Table 3: Phthalic acid catalyzed synthesis of 3,4-dihydropyrimidin-2-(1H)-one derivatives.

\begin{tabular}{|c|c|c|c|c|c|c|c|c|}
\hline Entry & $\mathrm{Ar}^{1}$ & $\mathrm{R}^{1}$ & $X$ & Product & Time (min) & Yield $\%^{a}$ & M.p. ${ }^{\circ} \mathrm{C}$ & Lit. M.p. ${ }^{\circ} \mathrm{C}$ \\
\hline 1 & $\mathrm{C}_{6} \mathrm{H}_{5}$ & $\mathrm{C}_{2} \mathrm{H}_{5}$ & $\mathrm{O}$ & $4 a$ & 15 & 85 & $200-202$ & $200-202^{20}$ \\
\hline 2 & $4-\mathrm{OH}-\mathrm{C}_{6} \mathrm{H}_{4}$ & $\mathrm{CH}_{3}$ & $\mathrm{O}$ & $4 b$ & 30 & 81 & $244-246$ & $245-246^{14}$ \\
\hline 3 & $4-\mathrm{Me}-\mathrm{C}_{6} \mathrm{H}_{4}$ & $\mathrm{C}_{2} \mathrm{H}_{5}$ & $\mathrm{O}$ & $4 c$ & 25 & 86 & 205-207 & $204-205^{14}$ \\
\hline 4 & $4-\mathrm{O}_{2} \mathrm{~N}-\mathrm{C}_{6} \mathrm{H}_{4}$ & $\mathrm{C}_{2} \mathrm{H}_{5}$ & $\mathrm{O}$ & $4 d$ & 20 & 89 & 209-211 & $211-213^{12}$ \\
\hline 5 & $3-\mathrm{Cl}-\mathrm{C}_{6} \mathrm{H}_{4}$ & $\mathrm{C}_{2} \mathrm{H}_{5}$ & $\mathrm{O}$ & $4 e$ & 35 & 83 & 189-191 & $191-193^{20}$ \\
\hline 6 & 4-OMe- $\mathrm{C}_{6} \mathrm{H}_{4}$ & $\mathrm{C}_{2} \mathrm{H}_{5}$ & $\mathrm{O}$ & $4 f$ & 25 & 84 & 203-204 & $203-205^{13}$ \\
\hline 7 & $4-\mathrm{F}-\mathrm{C}_{6} \mathrm{H}_{4}$ & $\mathrm{C}_{2} \mathrm{H}_{5}$ & $\mathrm{O}$ & $4 \mathrm{~g}$ & 15 & 87 & $176-178$ & $174-176^{18}$ \\
\hline 8 & $\mathrm{C}_{6} \mathrm{H}_{5}$ & $\mathrm{C}_{2} \mathrm{H}_{5}$ & $\mathrm{~S}$ & $4 h$ & 20 & 82 & $208-210$ & $208-210^{20}$ \\
\hline 9 & $4-\mathrm{Cl}-\mathrm{C}_{6} \mathrm{H}_{4}$ & $\mathrm{C}_{2} \mathrm{H}_{5}$ & $\mathrm{O}$ & $4 i$ & 35 & 85 & 214-216 & $214-215^{17}$ \\
\hline 10 & $4-\mathrm{O}_{2} \mathrm{~N}-\mathrm{C}_{6} \mathrm{H}_{4}$ & $\mathrm{CH}_{3}$ & $\mathrm{O}$ & $4 j$ & 15 & 90 & $216-218$ & $214-216^{16}$ \\
\hline 11 & $4-\mathrm{OH}-\mathrm{C}_{6} \mathrm{H}_{4}$ & $\mathrm{C}_{2} \mathrm{H}_{5}$ & $\mathrm{O}$ & $4 k$ & 35 & 78 & $232-234$ & $234-236^{12}$ \\
\hline 12 & $2-\mathrm{Cl}-\mathrm{C}_{6} \mathrm{H}_{4}$ & $\mathrm{C}_{2} \mathrm{H}_{5}$ & $\mathrm{O}$ & 41 & 35 & 81 & $218-220$ & $220-223^{20}$ \\
\hline 13 & 4-Cl- $\mathrm{C}_{6} \mathrm{H}_{4}$ & $\mathrm{C}_{2} \mathrm{H}_{5}$ & $\mathrm{~S}$ & $4 m$ & 45 & 79 & 191-193 & $191-195^{15}$ \\
\hline 14 & $2-\mathrm{Cl}-\mathrm{C}_{6} \mathrm{H}_{4}$ & $\mathrm{CH}_{3}$ & $\mathrm{O}$ & $4 n$ & 30 & 85 & $247-249$ & $248-252^{20}$ \\
\hline 15 & $4-\mathrm{F}-\mathrm{C}_{6} \mathrm{H}_{4}$ & $\mathrm{CH}_{3}$ & $\mathrm{~S}$ & 40 & 20 & 84 & $210-212$ & $208-210^{18}$ \\
\hline 16 & 4-OMe- $\mathrm{C}_{6} \mathrm{H}_{4}$ & $\mathrm{CH}_{3}$ & $\mathrm{O}$ & $4 p$ & 20 & 86 & 191-193 & $190-194^{16}$ \\
\hline
\end{tabular}

${ }^{a}$ Isolated yield.

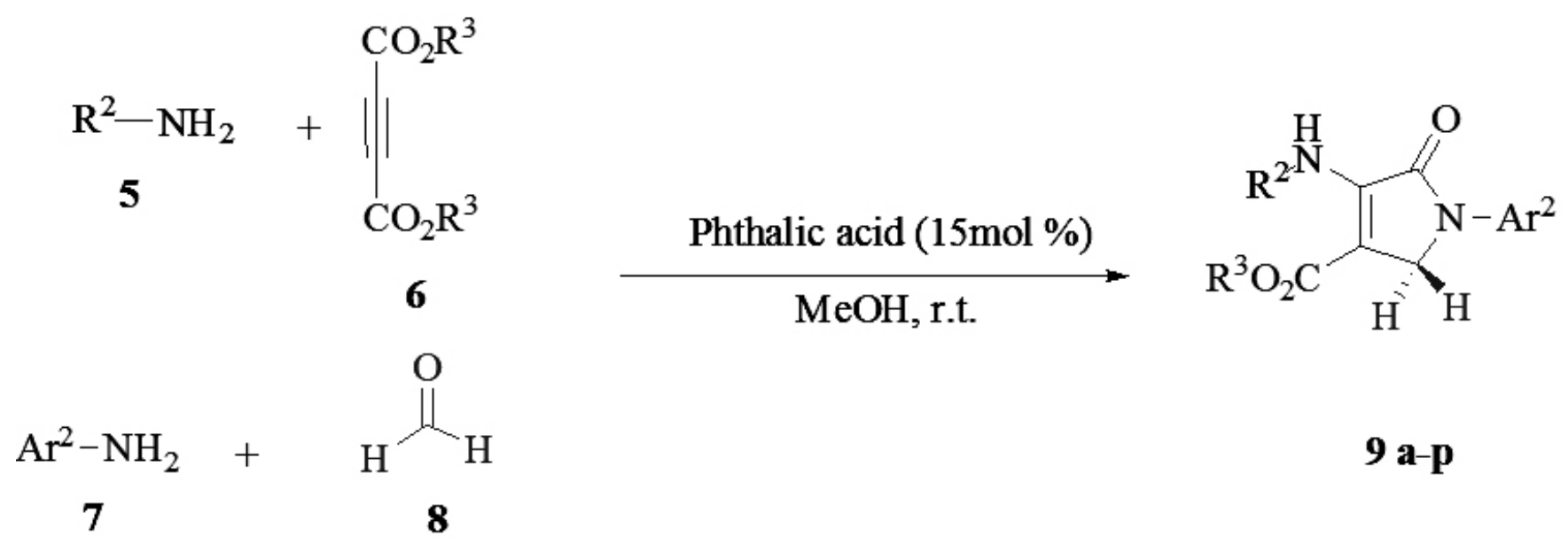

Scheme 2: Synthesis of substituted dihydro-2-oxypyrrole

The generality of this four condensation reaction was studied under optimized conditions and the reaction between aniline, dimethyl acetylenedicarboxylate (DMAD) and formaldehyde was investigation as a model reaction and then The effect of various solvents was investigated for this protocol $\mathrm{H}_{2} \mathrm{O}, \mathrm{EtOH}, \mathrm{MeOH}, \mathrm{CH}_{3} \mathrm{CN}, \mathrm{CHCl}_{3}, \mathrm{CH}_{2} \mathrm{Cl}_{2}$ and among these solvents, $\mathrm{MeOH}$ was found to be the best solvent for this methodology (Table 4 , entry 6) and the results are shown in Table 4. 
Table 4: Optimization of the reaction condition in the presence of different solvents ${ }^{a}$

\begin{tabular}{cccccc}
\hline & & & \\
\hline
\end{tabular}

${ }^{a}$ Reaction conditions: aniline $(2.0 \mathrm{mmol})$, dialkyl acetylenedicarboxylate $(1.0 \mathrm{mmol})$ and formaldehyde $(1.5 \mathrm{mmol})$ and catalyst in various solvents at room temperature.

The effect of different amount of catalyst was also studied in this protocol and in the absence of catalyst; a trace amount of this product was detected after $7 \mathrm{~h}$ (Table 5, entry 1). Good yields were obtained in the presence of catalyst. The best amount of catalyst was $15 \mathrm{~mol} \%(0.025 \mathrm{~g})$ (Table 5, entry 4$)$. The higher amount of catalyst did not increase the yields products (Table 5, entry 5) and the results are summarized in Table 5.

Table 5: Optimization of the reaction condition in the presence of different amounts of phthalic acid ${ }^{a}$

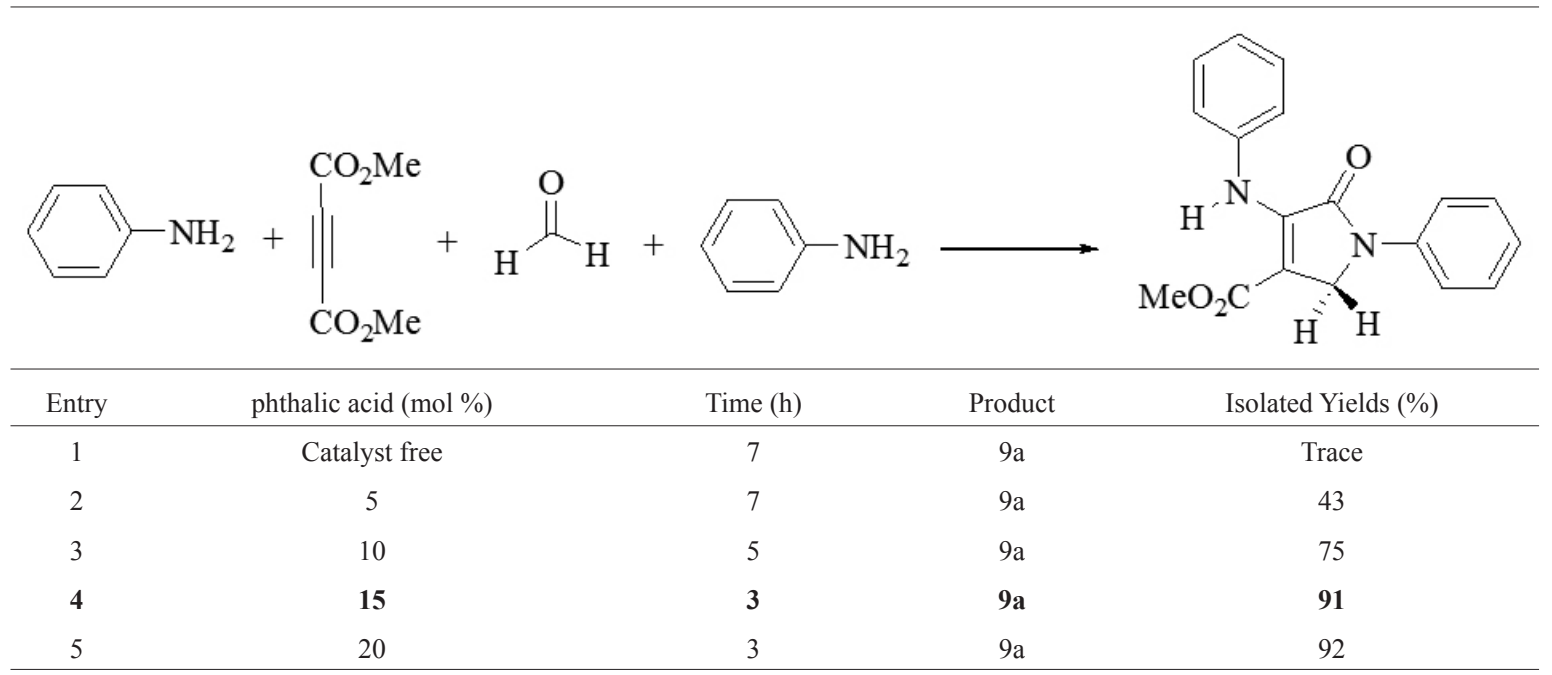

${ }^{a}$ Reaction conditions: aniline $(2.0 \mathrm{mmol})$, dialkyl acetylenedicarboxylate $(1.0 \mathrm{mmol})$ and formaldehyde $(1.5 \mathrm{mmol})$ and catalyst at room temperature.

Finally, we reported phthalic acid $(0.025 \mathrm{~g})$ as a biodegradable green and efficient catalyst for economical, environmental benign nature onepot four-component reaction of amines (aromatic or aliphatic), dialkyl acetylenedicarboxylate and formaldehyde in $\mathrm{MeOH}$ as solvent under ambient temperature.

In order to study of this procedure, we have synthesis a series of dihydro2-oxypyrrole derivatives with the type of aromatic or aliphatic amines with electron-donating or electron- withdrawing groups such as $\mathrm{Cl}, \mathrm{Br}, \mathrm{F}, \mathrm{Me}$, $\mathrm{OMe}, \ldots$ and dialkyl acetylenedicarboxylate with formaldehyde under ambient temperature in $\mathrm{MeOH}$ which gave excellent yields and the results are shown in Table 6 . The proposed mechanism for the synthesis of 3,4-dihydropyrimidin2-(1H)-one derivatives and dihydro-2-oxypyrroles are shown in scheme 3 and scheme 4 . 
Table 6: Phthalic acid catalyzed Synthesis of dihydro-2-oxypyrrole derivatives.

\begin{tabular}{|c|c|c|c|c|c|c|c|c|}
\hline Entry & $\mathrm{R}^{2}$ & $\mathrm{R}^{3}$ & $\mathrm{Ar}^{2}$ & Product & Time (h) & Yield $(\%)^{a}$ & M.p. ${ }^{\circ} \mathrm{C}$ & Lit. M.p. ${ }^{\circ} \mathrm{C}$ \\
\hline 1 & $\mathrm{Ph}$ & $\mathrm{Me}$ & $\mathrm{Ph}$ & $9 a$ & 3 & 91 & $155-157$ & $155-156^{21}$ \\
\hline 2 & $4-\mathrm{Br}-\mathrm{C}_{6} \mathrm{H}_{4}$ & $\mathrm{Me}$ & $4-\mathrm{Br}-\mathrm{C}_{6} \mathrm{H}_{4}$ & $9 b$ & 4.5 & 85 & $173-175$ & $175-177^{23}$ \\
\hline 3 & $4-\mathrm{F}-\mathrm{C}_{6} \mathrm{H}_{4}$ & $\mathrm{Me}$ & $4-\mathrm{F}-\mathrm{C}_{6} \mathrm{H}_{4}$ & $9 c$ & 2.5 & 94 & $164-166$ & $164-166^{26}$ \\
\hline 4 & $4-\mathrm{Me}-\mathrm{C}_{6} \mathrm{H}_{4}$ & $\mathrm{Me}$ & $4-\mathrm{Me}-\mathrm{C}_{6} \mathrm{H}_{4}$ & 9d & 3 & 88 & $178-179$ & $177-178^{21}$ \\
\hline 5 & $4-\mathrm{OMe}-\mathrm{C}_{6} \mathrm{H}_{4}$ & $\mathrm{Me}$ & $4-\mathrm{OMe}-\mathrm{C}_{6} \mathrm{H}_{4}$ & $9 e$ & 3 & 87 & $172-174$ & $172-175^{23}$ \\
\hline 6 & $\mathrm{PhCH}_{2}$ & $\mathrm{Me}$ & $\mathrm{Ph}$ & 9f & 3.5 & 92 & $139-141$ & $140-141^{22}$ \\
\hline 7 & $\mathrm{PhCH}_{2}$ & $\mathrm{Me}$ & $4-\mathrm{F}-\mathrm{C}_{6} \mathrm{H}_{4}$ & $9 \mathrm{~g}$ & 3 & 93 & $164-166$ & $166-168^{24}$ \\
\hline 8 & $\mathrm{n}-\mathrm{C}_{4} \mathrm{H}_{9}$ & $\mathrm{Me}$ & $\mathrm{Ph}$ & $9 h$ & 2.5 & 93 & $60-62$ & $60^{21}$ \\
\hline 9 & $\mathrm{n}-\mathrm{C}_{4} \mathrm{H}_{9}$ & $\mathrm{Me}$ & 4- $\mathrm{Br}-\mathrm{C}_{6} \mathrm{H}_{4}$ & $9 \mathrm{i}$ & 3 & 89 & $174-176$ & $175-177^{23}$ \\
\hline 10 & $\mathrm{Ph}$ & Et & $\mathrm{Ph}$ & $9 j$ & 3 & 89 & $139-141$ & $138-140^{22}$ \\
\hline 11 & 4- $\mathrm{Br}-\mathrm{C}_{6} \mathrm{H}_{4}$ & Et & 4- $\mathrm{Br}-\mathrm{C}_{6} \mathrm{H}_{4}$ & $9 \mathbf{k}$ & 5 & 84 & $170-172$ & $169-171^{22}$ \\
\hline 12 & $4-\mathrm{F}-\mathrm{C}_{6} \mathrm{H}_{4}$ & Et & $4-\mathrm{F}-\mathrm{C}_{6} \mathrm{H}_{4}$ & 91 & 3 & 90 & $174-176$ & $172-174^{23}$ \\
\hline 13 & 4-Me- $\mathrm{C}_{6} \mathrm{H}_{4}$ & Et & $4-\mathrm{Me}-\mathrm{C}_{6} \mathrm{H}_{4}$ & $9 m$ & 4 & 85 & $131-133$ & $131-132^{22}$ \\
\hline 14 & $4-\mathrm{OMe}-\mathrm{C}_{6} \mathrm{H}_{4}$ & Et & $4-\mathrm{OMe}-\mathrm{C}_{6} \mathrm{H}_{4}$ & $9 n$ & 3 & 84 & $153-155$ & $153-155^{26}$ \\
\hline 15 & $\mathrm{PhCH}_{2}$ & Et & $\mathrm{Ph}$ & 90 & 4 & 89 & $131-133$ & $130-132^{22}$ \\
\hline 16 & $n-\mathrm{C}_{4} \mathrm{H}_{9}$ & Et & $4-\mathrm{Br}^{-} \mathrm{C}_{6} \mathrm{H}_{4}$ & $9 p$ & 3 & 86 & $93-95$ & $94-96^{26}$ \\
\hline
\end{tabular}

${ }^{a}$ Isolated yield.<smiles>[X]C(N)=[C+]C(=O)[Al]</smiles>

1<smiles>CC(=O)CC(=O)O[Ga]</smiles>

3<smiles>[X]C(N)=NC=[W]</smiles>

$\mathbf{A}$<smiles>[Y]C(N)NC([Al])C(C(C)=O)C(=O)O[R4]([H])=C</smiles>

C<smiles>[R4]OC(=O)C1=C(C)NC([X])NC1[Al]</smiles>

B

Scheme 3: Proposed mechanistic route for the synthesis of 3,4-dihydropyrimidin-2-( $1 H)$-ones

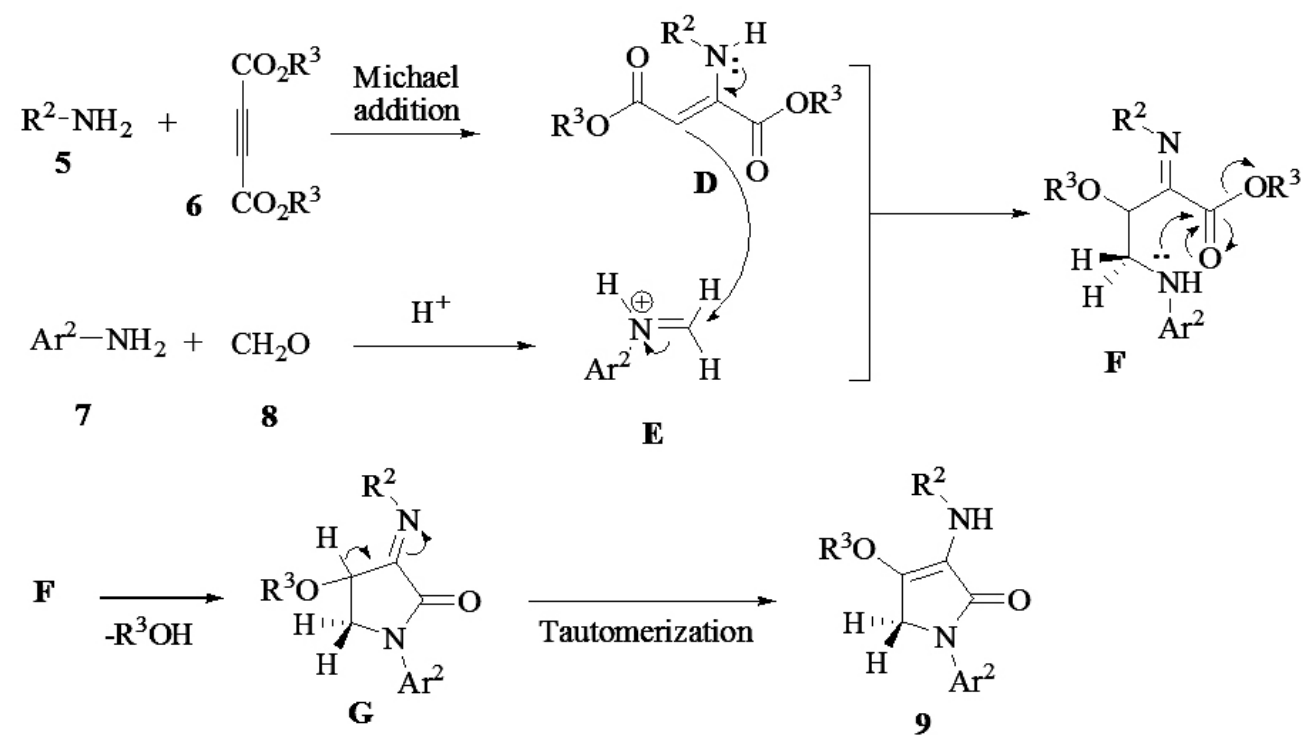

Scheme 4: Proposed mechanistic route for the synthesis of substituted dihydro-2-oxypyrrole 
Comparison of catalytic ability some of catalysts reported in the literature for synthesis of 3,4-dihydropyrimidin-2-( $1 H)$-one derivatives are shown in Table 7 and substituted dihydro-2-oxypyrrole are shown in Table 8.

Table 7: Comparison of catalytic ability some of catalysts reported in the literature for synthesis of 3, 4-dihydropyrimidin-2-(1H)-ones derivatives ${ }^{a}$

\begin{tabular}{|c|c|c|c|c|}
\hline Entry & Catalyst & Conditions & Time/Yield (\%) & Refrences \\
\hline 1 & bakers yeast & Room temperature & $24 \mathrm{~h} / 84$ & {$[14]$} \\
\hline 2 & $\mathrm{Cu}\left(\mathrm{BF}_{4}\right)_{2} \cdot \mathrm{xH}_{2} \mathrm{O}$ & Room temperature & $30 \mathrm{~min} / 90$ & {$[16]$} \\
\hline 3 & Hydrotalcite & Solvent-free, $80{ }^{\circ} \mathrm{C}$ & $35 \mathrm{~min} / 84$ & {$[17]$} \\
\hline 4 & {$\left[\mathrm{Al}\left(\mathrm{H}_{2} \mathrm{O}\right)_{6}\right]\left(\mathrm{BF}_{4}\right)_{3}$} & MeCN, Reflux & $20 \mathrm{~h} / 81$ & [19] \\
\hline
\end{tabular}

${ }^{a}$ Based on the three-component reaction of benzaldehyde, ethyl acetoacetate and urea.

Table 8: Comparison of catalytic ability some of catalysts reported in the literature for synthesis of substituted dihydro-2-oxypyrrole ${ }^{a}$

\begin{tabular}{|c|c|c|c|c|}
\hline Entry & Catalyst & Conditions & Time/Yield (\%) & Refrences \\
\hline 1 & $\mathrm{I}_{2}$ & $\mathrm{MeOH}$, r.t. & $1 \mathrm{~h} / 82$ & {$[21]$} \\
\hline 2 & {$\left[\mathrm{n}-\mathrm{Bu}_{4} \mathrm{~N}\right]\left[\mathrm{HSO}_{4}\right]$} & $\mathrm{MeOH}$, r.t. & $4 \mathrm{~h} / 88$ & {$[23]$} \\
\hline 3 & $\mathrm{ZrCl}_{4}$ & MeOH, r.t. & $4 \mathrm{~h} / 84$ & {$[26]$} \\
\hline 4 & $\mathrm{InCl}_{3}$ & $\mathrm{MeOH}$, r.t. & $3 \mathrm{~h} / 85$ & [27] \\
\hline
\end{tabular}

${ }^{a}$ Based on the four-component reaction of aniline, dimethylacetylenedicarboxylate, formaldehyde.

\section{EXPERIMENTAL}

\section{General}

Melting points and IR spectra all compounds were determined using an Electro thermal 9100 apparatus and a JASCO FTIR 460 Plus spectrometer. Also, nuclear magnetic resonance, ${ }^{1} \mathrm{H}$ NMR spectra were recorded on a Bruker DRX-400 Avance instruments with DMSO- $\mathrm{d}_{6}$ and $\mathrm{CDCl}_{3}$ as solvents. In the present literature, all reagents and solvents were purchased from Merck, Fluka and Acros chemical companies were used without further purification.

General procedure for preparation of 3,4-dihydropyrimidin-2-(1H)ones derivatives $4 \mathbf{a}-\mathbf{p}$

A mixture of aldehydes derivatives $(\mathbf{1}, 1.0 \mathrm{mmol})$ and urea/thiourea $(\mathbf{2}, 1.5$ $\mathrm{mmol})$, ethyl/methyl acetoacetate $(\mathbf{3}, 1.0 \mathrm{mmol})$ under solvent-free conditions was heated for appropriate time in the presence of phthalic acid $(15 \mathrm{~mol} \%)$ at $80^{\circ} \mathrm{C}$. After completion of the reaction (by thin layer chromatography TLC) the mixture was cooled to rt and cold water was added and the precipitated was separated with filtration and solid was recrystallized from ethanol to afford the pure products (4a- p). All products were characterized by comparison of spectroscopic data $\left({ }^{1} \mathrm{HNMR}\right)$. Spectra data of selected and known products are represented below: (4a)

5-Ethoxycarbonyl-6-methyl-4-phenyl-3,4-dihydropyrimidin-2(1H)-one

Crystalline solid, mp: $200-202{ }^{\circ} \mathrm{C} ;{ }^{1} \mathrm{H}$ NMR (400 MHz, DMSO-d $): 1.10$ $\left(3 \mathrm{H}, \mathrm{t}, J=7.2 \mathrm{~Hz}, \mathrm{CH}_{3} \mathrm{CH}_{2}\right), 2.26\left(3 \mathrm{H}, \mathrm{s}, \mathrm{CH}_{3}\right), 3.99\left(2 \mathrm{H}, \mathrm{q}, J=7.2 \mathrm{~Hz}, \mathrm{CH}_{2} \mathrm{O}\right)$ $5.15(1 \mathrm{H}, \mathrm{s}, \mathrm{CHN}), 7.26(3 \mathrm{H}, \mathrm{d}, J=7.2 \mathrm{~Hz}, \mathrm{ArH}), 7.33(2 \mathrm{H}, \mathrm{t}, J=7.2 \mathrm{~Hz}, \mathrm{ArH})$ 7.76 and $9.21(2 \mathrm{H}, 2 \mathrm{~s}, 2 \mathrm{NH})$.

5-Methoxycarbonyl-6-methyl-4-(4-hydroxyphenyl)-3,4-dihydropyrimidin2(1H)-one $(\mathbf{4 b})$

Crystalline solid, mp: $244-246{ }^{\circ} \mathrm{C} ;{ }^{1} \mathrm{H}$ NMR (400 MHz, DMSO-d $): 2.31$ $\left(3 \mathrm{H}, \mathrm{s}, \mathrm{CH}_{3}\right), 3.46(3 \mathrm{H}, \mathrm{s}, \mathrm{OCH}), 5.04(1 \mathrm{H}, \mathrm{s}, \mathrm{CHN}), 6.70(2 \mathrm{H}, \mathrm{d}, J=8.8 \mathrm{~Hz}$ $\mathrm{ArH}), 7.02(2 \mathrm{H}, \mathrm{d}, J=9.6 \mathrm{~Hz}, \mathrm{ArH}), 7.67$ and $9.16(2 \mathrm{H}, 2 \mathrm{~s}, 2 \mathrm{NH}), 9.36(1 \mathrm{H}, \mathrm{s}$, $\mathrm{OH})$.

5-Ethoxycarbonyl-6-methyl-4-(4-methylphenyl)-3,4-dihydropyrimidin2(1H)-one $(4 c)$

Crystalline solid, mp: $205-207{ }^{\circ} \mathrm{C} ;{ }^{1} \mathrm{H}$ NMR (400 MHz, DMSO-d $): 1.11$ $\left(3 \mathrm{H}, \mathrm{t}, J=7.2 \mathrm{~Hz}, \mathrm{CH}_{3} \mathrm{CH}_{2}\right), 2.26\left(6 \mathrm{H}, \mathrm{d}, J=9.2 \mathrm{~Hz}, 2 \mathrm{CH}_{3}\right), 3.99(2 \mathrm{H}, \mathrm{q}, J=7.2$ $\left.\mathrm{Hz}, \mathrm{CH}_{2} \mathrm{O}\right), 5.11(1 \mathrm{H}, \mathrm{s}, \mathrm{CHN}), 7.13(4 \mathrm{H}, \mathrm{s}, \mathrm{ArH}), 7.70$ and $9.17(2 \mathrm{H}, 2 \mathrm{~s}, 2 \mathrm{NH})$.

5-Ethoxycarbonyl-6-methyl-4-(4-nitrophenyl)-3,4-dihydropyrimidin2(1H)-one (4d) Crystalline solid, mp: 209-211 ${ }^{\circ} \mathrm{C}$; ${ }^{1} \mathrm{H}$ NMR $(400 \mathrm{MHz}$ DMSO-d 6 ): $1.10\left(3 \mathrm{H}, \mathrm{t}, J=9.6 \mathrm{~Hz}, \underline{\mathrm{CH}}_{3} \mathrm{CH}_{2}\right), 2.28\left(3 \mathrm{H}, \mathrm{s}, \mathrm{CH}_{3}\right), 3.99(2 \mathrm{H}, \mathrm{q}$, $\left.J=9.2 \mathrm{~Hz}, \mathrm{CH}_{2} \mathrm{O}\right), 5.27(1 \mathrm{H}, \mathrm{s}, \mathrm{CHN}), 7.50-7.53(2 \mathrm{H}, \mathrm{m}, \mathrm{ArH}), 7.23(2 \mathrm{H}, \mathrm{d}, J=$
9. $2 \mathrm{~Hz}, \mathrm{ArH}), 7.92$ and $9.38(2 \mathrm{H}, 2 \mathrm{~s}, 2 \mathrm{NH})$

5-Ethoxycarbonyl-6-methyl-4-(3-chlorophenyl)-3,4-dihydropyrimidin2(1H)-one (4e) Crystalline solid, mp: 189-191 ${ }^{\circ} \mathrm{C} ;{ }^{1} \mathrm{H}$ NMR $(400 \mathrm{MHz}$, DMSO-d $)_{6}: 1.11\left(3 \mathrm{H}, \mathrm{t}, J=9.2 \mathrm{~Hz}, \mathrm{CH}_{3} \mathrm{CH}_{2}\right), 2.26\left(3 \mathrm{H}, \mathrm{s}, \mathrm{CH}_{3}\right), 4.01(2 \mathrm{H}, \mathrm{q}$ $\left.J=9.6 \mathrm{~Hz}, \mathrm{CH}_{2} \mathrm{O}\right), 5.15(1 \mathrm{H}, \mathrm{s}, \mathrm{CHN}), 7.19-7.26(2 \mathrm{H}, \mathrm{m}, \mathrm{ArH}), 7.31-7.41(2 \mathrm{H}$ $\mathrm{m}, \mathrm{ArH}), 7.83$ and $9.30(2 \mathrm{H}, 2 \mathrm{~s}, 2 \mathrm{NH})$

5-Ethoxycarbonyl-6-methyl-4-(4-methoxyphenyl)-3,4-dihydropyrimidin2(1H)-one (4f)

Crystalline solid, mp: $203-204^{\circ} \mathrm{C} ;{ }^{1} \mathrm{H}$ NMR (400 MHz, DMSO-d ): 1.11 $\left(3 \mathrm{H}, \mathrm{t}, J=9.6 \mathrm{~Hz}, \mathrm{CH}_{3} \mathrm{CH}_{2}\right), 2.24\left(3 \mathrm{H}, \mathrm{s}, \mathrm{CH}_{3}\right), 3.73\left(3 \mathrm{H}, \mathrm{s}, \mathrm{OCH}_{3}\right), 3.99(2 \mathrm{H}$ q, $\left.J=9.6 \mathrm{~Hz}, \mathrm{CH}_{2} \mathrm{O}\right), 5.09(1 \mathrm{H}, \mathrm{s}, \mathrm{CHN}), 6.89(2 \mathrm{H}, \mathrm{d}, J=8.4 \mathrm{~Hz}, \mathrm{ArH}), 7.15(2 \mathrm{H}$, $\mathrm{d}, J=8.8 \mathrm{~Hz}, \mathrm{ArH}), 7.70$ and $9.18(2 \mathrm{H}, 2 \mathrm{~s}, 2 \mathrm{NH})$.

5-Ethoxycarbonyl-6-methyl-4-(4-fluorophenyl)-3,4-dihydropyrimidin2(1H)-one (4g) Crystalline solid, mp: $176-178^{\circ} \mathrm{C} ;{ }^{1} \mathrm{H}$ NMR $(400 \mathrm{MHz}$, DMSO-d $)_{6}: 1.11\left(3 \mathrm{H}, \mathrm{t}, J=9.6 \mathrm{~Hz}, \mathrm{CH}_{3} \mathrm{CH}_{2}\right), 2.25\left(3 \mathrm{H}, \mathrm{s}, \mathrm{CH}_{3}\right), 3.99(2 \mathrm{H}, \mathrm{q}$, $\left.J=9.6 \mathrm{~Hz}, \mathrm{CH}_{2} \mathrm{O}\right), 5.14(1 \mathrm{H}, \mathrm{s}, \mathrm{CHN}), 7.13-7.20(2 \mathrm{H}, \mathrm{m}, \mathrm{ArH}), 7.24-7.29(2 \mathrm{H}$, $\mathrm{m}, \mathrm{ArH}), 7.78$ and $9.25(2 \mathrm{H}, 2 \mathrm{~s}, 2 \mathrm{NH})$ (4h)

5-Ethoxycarbonyl-6-methyl-4-phenyl-3,4-dihydropyrimidin-2(1H)-thione

Crystalline solid, mp: $208-210{ }^{\circ} \mathrm{C} ;{ }^{1} \mathrm{H}$ NMR (400 MHz, DMSO-d ): 1.11 $\left(3 \mathrm{H}, \mathrm{t}, J=7.2 \mathrm{~Hz}, \mathrm{CH}_{3} \mathrm{CH}_{2}\right), 2.31\left(3 \mathrm{H}, \mathrm{s}, \mathrm{CH}_{3}\right), 4.02\left(2 \mathrm{H}, \mathrm{q}, J=7.2 \mathrm{~Hz}, \mathrm{CH}_{2} \mathrm{O}\right)$ $5.19(1 \mathrm{H}, \mathrm{s}, \mathrm{CHN}), 7.23(2 \mathrm{H}, \mathrm{d}, J=7.2 \mathrm{~Hz}, \mathrm{ArH}), 7.28(1 \mathrm{H}, \mathrm{t}, J=7.2 \mathrm{~Hz}, \mathrm{ArH})$ $7.36(2 \mathrm{H}, \mathrm{t}, J=7.2 \mathrm{~Hz}, \mathrm{ArH}), 9.68$ and $10.36(2 \mathrm{H}, 2 \mathrm{~s}, 2 \mathrm{NH})$.

5-Ethoxycarbonyl-6-methyl-4-(4-chlorophenyl)-3,4-dihydropyrimidin2(1H)-one (4i) Crystalline solid, mp: 214-216 ${ }^{\circ} \mathrm{C} ;{ }^{1} \mathrm{H}$ NMR $(400 \mathrm{MHz}$ DMSO-d $): 1.10\left(3 \mathrm{H}, \mathrm{t}, J=9.2 \mathrm{~Hz}, \mathrm{CH}_{3} \mathrm{CH}_{2}\right), 2.25\left(3 \mathrm{H}, \mathrm{s}, \mathrm{CH}_{3}\right), 3.99(2 \mathrm{H}, \mathrm{q}$ $\left.J=9.2 \mathrm{~Hz}, \mathrm{CH}_{2} \mathrm{O}\right), 5.14(1 \mathrm{H}, \mathrm{s}, \mathrm{CHN}), 7.25(2 \mathrm{H}, \mathrm{d}, J=11.2 \mathrm{~Hz}, \mathrm{ArH}), 7.39(2 \mathrm{H}$ $\mathrm{d}, J=11.2 \mathrm{~Hz}, \mathrm{ArH}), 7.79$ and $9.27(2 \mathrm{H}, 2 \mathrm{~s}, 2 \mathrm{NH})$.

5-Methoxycarbonyl-6-methyl-4-(4-nitrophenyl)-3,4-dihydropyrimidin2(1H)-one (4j)

Crystalline solid, mp: $216-218{ }^{\circ} \mathrm{C} ;{ }^{1} \mathrm{H}$ NMR (400 MHz, DMSO-d ): 2.2 $8\left(3 \mathrm{H}, \mathrm{s}, \mathrm{CH}_{3}\right), 3.55\left(3 \mathrm{H}, \mathrm{s}, \mathrm{OCH}_{3}\right), 5.28(1 \mathrm{H}, \mathrm{s}, \mathrm{CHN}), 7.52(2 \mathrm{H}, \mathrm{d}, J=8.4 \mathrm{~Hz}$ $\mathrm{ArH}), 7.22(2 \mathrm{H}, \mathrm{d}, J=8.8 \mathrm{~Hz}, \mathrm{ArH}), 7.93$ and $9.40(2 \mathrm{H}, 2 \mathrm{~s}, 2 \mathrm{NH})$.

5-Ethoxycarbonyl-6-methyl-4-(4-hydroxyphenyl)-3,4-dihydropyrimidin2(1H)-one (4k)

Crystalline solid, mp: $232-234{ }^{\circ} \mathrm{C} ;{ }^{1} \mathrm{H}$ NMR (400 MHz, DMSO-d ): 1.11 $\left(3 \mathrm{H}, \mathrm{t}, J=9.6 \mathrm{~Hz}, \mathrm{CH}_{3} \mathrm{CH}_{2}\right), 2.50\left(3 \mathrm{H}, \mathrm{s}, \mathrm{CH}_{3}\right), 3.98\left(2 \mathrm{H}, \mathrm{q}, J=9.2 \mathrm{~Hz}, \mathrm{CH}_{2} \mathrm{O}\right)$ $5.04(1 \mathrm{H}, \mathrm{s}, \mathrm{CHN}), 6.68-7.04(4 \mathrm{H}, \mathrm{m}, \mathrm{ArH}), 7.64$ and $9.13(2 \mathrm{H}, 2 \mathrm{~s}, 2 \mathrm{NH}), 9.35$ $(1 \mathrm{H}, \mathrm{s}, \mathrm{OH})$.

5-Ethoxycarbonyl-6-methyl-4-(2-chlorophenyl)-3,4-dihydropyrimidin2(1H)-one (4l) Crystalline solid, mp: $218-220{ }^{\circ} \mathrm{C}$; ${ }^{1} \mathrm{H}$ NMR $(400 \mathrm{MHz}$ DMSO-d 6$): 1.00\left(3 \mathrm{H}, \mathrm{t}, J=9.2 \mathrm{~Hz}, \underline{\mathrm{CH}}_{3} \mathrm{CH}_{2}\right), 2.31\left(3 \mathrm{H}, \mathrm{s}, \mathrm{CH}_{3}\right), 4.02(2 \mathrm{H}, \mathrm{q}$ 
$\left.J=9.2 \mathrm{~Hz}, \mathrm{CH}_{2} \mathrm{O}\right), 5.63(1 \mathrm{H}, \mathrm{s}, \mathrm{CHN}), 7.25-7.34(3 \mathrm{H}, \mathrm{m}, \mathrm{ArH}), 7.41(1 \mathrm{H}, \mathrm{d}$, $J=8.8 \mathrm{~Hz}, \mathrm{ArH}), 7.73$ and $9.29(2 \mathrm{H}, 2 \mathrm{~s}, 2 \mathrm{NH})$

5-Methoxycarbonyl-6-methyl-4-(2-chlorophenyl)-3,4-dihydropyrimidin2(1H)-one (4n)

Crystalline solid, mp: $247-249{ }^{\circ} \mathrm{C} ;{ }^{1} \mathrm{H}$ NMR (400 MHz, DMSO-d $): 2.31$ $\left(3 \mathrm{H}, \mathrm{s}, \mathrm{CH}_{3}\right), 3.46\left(3 \mathrm{H}, \mathrm{s}, \mathrm{OCH}_{3}\right), 5.62(1 \mathrm{H}, \mathrm{s}, \mathrm{CHN}), 7.28-7.34(3 \mathrm{H}, \mathrm{m}, \mathrm{ArH})$, $7.42(1 \mathrm{H}, \mathrm{d}, J=7.2 \mathrm{~Hz}, \mathrm{ArH}), 7.72$ and $9.36(2 \mathrm{H}, 2 \mathrm{~s}, 2 \mathrm{NH})$.

5-Methoxycarbonyl-6-methyl-4-(4-fluorophenyl)-3,4-dihydropyrimidin2(1H)-thione (4o)

Crystalline solid, mp: $210-212{ }^{\circ} \mathrm{C} ;{ }^{1} \mathrm{H}$ NMR (400 MHz, DMSO-d ) : 2.30 $\left(3 \mathrm{H}, \mathrm{s}, \mathrm{CH}_{3}\right), 3.56\left(3 \mathrm{H}, \mathrm{s}, \mathrm{OCH}_{3}\right), 5.18(1 \mathrm{H}, \mathrm{s}, \mathrm{CHN}), 7.13-7.28(4 \mathrm{H}, \mathrm{m}, \mathrm{ArH})$, 9.71 and $10.42(2 \mathrm{H}, 2 \mathrm{~s}, 2 \mathrm{NH})$.

5-Methoxycarbonyl-6-methyl-4-(4-methoxyphenyl)-3,4-dihydropyrimidin2(1H)-one $(4 p)$

Crystalline solid, mp: $191-193^{\circ} \mathrm{C} ;{ }^{1} \mathrm{H}$ NMR (400 MHz, DMSO-d ): 2.25 $\left(3 \mathrm{H}, \mathrm{s}, \mathrm{CH}_{3}\right), 3.53\left(3 \mathrm{H}, \mathrm{s}, \mathrm{OCH}_{3}\right), 3.73\left(3 \mathrm{H}, \mathrm{s}, \mathrm{OCH}_{3}\right), 5.09(1 \mathrm{H}, \mathrm{s}, \mathrm{CHN}), 6.88$ $(2 \mathrm{H}, \mathrm{d}, J=8.8 \mathrm{~Hz}, \mathrm{ArH}), 7.14(2 \mathrm{H}, \mathrm{d}, J=8.8 \mathrm{~Hz}, \mathrm{ArH}), 7.71$ and $9.20(2 \mathrm{H}, 2 \mathrm{~s}$, $2 \mathrm{NH})$.

General procedure for preparation of substituted dihydro-2oxypyrroles 9a-p

A mixture of amine $(\mathbf{5}, 1.0 \mathrm{mmol})$ and dialkyl acetylenedicarboxylate $(\mathbf{6}$, $1.0 \mathrm{mmol}$ ) was stirred in $\mathrm{MeOH}(3 \mathrm{~mL})$ for $15 \mathrm{~min}$. next, amine $(7,1.0 \mathrm{mmol})$ and formaldehyde $(8,1.5 \mathrm{mmol})$ and phthalic acid $(0.025 \mathrm{~g})$ were added and the reaction was stirred for appropriate time. After completion of the reaction (by thin layer chromatography TLC), the mixture was separated with filtration and the solid washed with ethanol $(3 \times 2 \mathrm{~mL})$ with no column chromatographic separation to give pure compounds (9a-p). All products were characterized by comparison of spectroscopic data (FT-IR, ${ }^{1} \mathrm{HNMR}$ ). Spectra data of selected and known products are represented below:

Methyl 2,5-dihydro-2-oxo-1-phenyl-3-(phenylamino)-1H-pyrrole4carboxylate $(9 a)$

Solid powder, mp: $155-157^{\circ} \mathrm{C}$; IR $\left(\mathrm{KBr}, \mathrm{cm}^{-1}\right)$ : v $3264(\mathrm{NH}), 1692(\mathrm{C}=\mathrm{O})$, $1641(\mathrm{C}=\mathrm{O}) ;{ }^{1} \mathrm{H}$ NMR $\left(400 \mathrm{MHz}, \mathrm{CDCl}_{3}\right): \delta 3.76\left(3 \mathrm{H}, \mathrm{s}, \mathrm{OCH}_{3}\right), 4.57(2 \mathrm{H}$, s, $\left.\mathrm{CH}_{2}-\mathrm{N}\right), 7.16-7.23(4 \mathrm{H}, \mathrm{m}, \mathrm{ArH}), 7.35(2 \mathrm{H}, \mathrm{t}, J=7.8 \mathrm{~Hz}, \mathrm{ArH}), 7.42(2 \mathrm{H}, \mathrm{t}$, $J=7.8 \mathrm{~Hz}, \mathrm{ArH}), 7.81(2 \mathrm{H}, \mathrm{d}, J=8.0 \mathrm{~Hz}, \mathrm{ArH}), 8.05(1 \mathrm{H}, \mathrm{s}, \mathrm{NH})$.

Methyl4-(4-bromophenylamino)-1-(4-bromophenyl) 2,5-dihydro-5-oxo1 H-pyrrole-3-carboxylate (9b)

Solid powder, mp: $173-175{ }^{\circ} \mathrm{C} ;{ }^{1} \mathrm{HNMR}\left(400 \mathrm{MHz}, \mathrm{CDCl}_{3}\right): \delta 3.78(3 \mathrm{H}$, $\left.\mathrm{s}, \mathrm{OCH}_{3}\right), 4.50\left(2 \mathrm{H}, \mathrm{s}, \mathrm{CH}_{2}-\mathrm{N}\right), 7.08(2 \mathrm{H}, \mathrm{d}, J=8.8 \mathrm{~Hz}, \mathrm{ArH}), 7.30(2 \mathrm{H}, \mathrm{d}, J=$ $8.4 \mathrm{~Hz}, \mathrm{ArH}), 7.35(2 \mathrm{H}, \mathrm{d}, J=8.8 \mathrm{~Hz}, \mathrm{ArH}), 7.72(2 \mathrm{H}, \mathrm{d}, J=8.8 \mathrm{~Hz}, \mathrm{ArH}), 8.03$ $(1 \mathrm{H}, \mathrm{s}, \mathrm{NH})$.

Methyl 4-(4-fluoroyphenylamino)-1-(4-fluorophenyl)-2, 5-dihydro-5-oxo1 H-pyrrole-3-carboxylate $(\mathbf{9 c})$

Solid powder, mp: $164-166{ }^{\circ} \mathrm{C} ;{ }^{1} \mathrm{H}$ NMR $\left(400 \mathrm{MHz}, \mathrm{CDCl}_{3}\right): 3.79(3 \mathrm{H}, \mathrm{s}$, $\left.\mathrm{OCH}_{3}\right), 4.52\left(2 \mathrm{H}, \mathrm{s}, \mathrm{CH}_{2}-\mathrm{N}\right), 7.04(2 \mathrm{H}, \mathrm{t}, J=8.4 \mathrm{~Hz}, \mathrm{ArH}), 7.08-7.16(4 \mathrm{H}, \mathrm{m}$, ArH) $7.73-7.79(2 \mathrm{H}, \mathrm{m}, \mathrm{ArH}), 8.05(1 \mathrm{H}, \mathrm{s}, \mathrm{NH})$

Methyl 4-(4-methylphenylamino)-1-(4-methylphenyl)-2, 5-dihydro-5-oxo-

1H-pyrrole-3-carboxylate (9d)

Solid powder, mp: $178-179^{\circ} \mathrm{C} ;{ }^{1} \mathrm{H}$ NMR $\left(400 \mathrm{MHz}, \mathrm{CDCl}_{3}\right): 2.36(3 \mathrm{H}, \mathrm{s}$, $\left.2 \mathrm{CH}_{3}\right), 3.77\left(3 \mathrm{H}, \mathrm{s}, \mathrm{OCH}_{3}\right), 4.52\left(2 \mathrm{H}, \mathrm{s}, \mathrm{CH}_{2}-\mathrm{N}\right), 7.06(2 \mathrm{H}, \mathrm{d}, J=8.4 \mathrm{~Hz}, \mathrm{ArH})$, $7.14(2 \mathrm{H}, \mathrm{d}, J=8.4 \mathrm{~Hz}, \mathrm{ArH}), 7.21(2 \mathrm{H}, \mathrm{d}, J=8.4 \mathrm{~Hz}, \mathrm{ArH}), 7.68(2 \mathrm{H}, \mathrm{d}, J=8.8$ $\mathrm{Hz}, \mathrm{ArH}), 8.03$ (1H, s, NH).

Methyl 4-(4-methoxyphenylamino)-1-(4-methoxyphenyl)-2, 5-dihydro-5oxo-1H-pyrrole-3-carboxylate $(\mathbf{9 e})$

Solid powder, mp: $172-174{ }^{\circ} \mathrm{C} ;{ }^{1} \mathrm{H}$ NMR $\left(400 \mathrm{MHz}, \mathrm{CDCl}_{3}\right): 3.77(3 \mathrm{H}, \mathrm{s}$, $\left.\mathrm{CH}_{3}\right), 3.83\left(6 \mathrm{H}, \mathrm{s}, 2 \mathrm{OCH}_{3}\right), 4.50\left(2 \mathrm{H}, \mathrm{s}, \mathrm{CH}_{2}-\mathrm{N}\right), 6.89(4 \mathrm{H}, \mathrm{d}, J=17.6 \mathrm{~Hz}, \mathrm{ArH})$, $7.13(1 \mathrm{H}, \mathrm{s}, \mathrm{ArH}), 7.68(1 \mathrm{H}, \mathrm{s}, \mathrm{ArH}), 8.03(1 \mathrm{H}, \mathrm{s}, \mathrm{NH})$

Methyl 3-(benzylamino)-1-phenyl-2,5-dihydro-2-oxo-1H-pyrrole-4carboxylate (9f)

Solid powder, mp: $139-141{ }^{\circ} \mathrm{C}$; ${ }^{1} \mathrm{H}$ NMR $\left(400 \mathrm{MHz}, \mathrm{CDCl}_{3}\right): \delta 3.80(\mathrm{~s}, 3 \mathrm{H}$, $\left.\mathrm{OCH}_{3}\right), 4.48\left(\mathrm{~s}, 2 \mathrm{H}, \underline{\mathrm{CH}}_{2}-\mathrm{N}\right), 5.10\left(\mathrm{~d}, 2 \mathrm{H}, J=6.4 \mathrm{~Hz}, \underline{\mathrm{CH}}_{2}-\mathrm{NH}\right), 6.90(\mathrm{br}, 1 \mathrm{H}$, $\mathrm{NH}), 7.18-7.41(\mathrm{~m}, 8 \mathrm{H}, \mathrm{ArH}), 7.73(\mathrm{~d}, 2 \mathrm{H}, J=8.0 \mathrm{~Hz}, \mathrm{ArH})$.

Methyl 3-(benzylamino)-1-(4-fluorophenyl)-2,5-dihydro-2-oxo-1Hpyrrole-4-carboxylate $(\mathbf{9 g})$

Solid powder, mp: $164-166^{\circ} \mathrm{C}$; IR $\left(\mathrm{KBr}, \mathrm{cm}^{-1}\right)$ : v $3320(\mathrm{NH}), 1697(\mathrm{C}=\mathrm{O})$, $1645(\mathrm{C}=\mathrm{O}) ;{ }^{1} \mathrm{H}$ NMR $\left(400 \mathrm{MHz}, \mathrm{CDCl}_{3}\right): \delta 3.80\left(\mathrm{~s}, 3 \mathrm{H}, \mathrm{OCH}_{3}\right), 4.42(\mathrm{~s}, 2 \mathrm{H}$, $\left.\mathrm{CH}_{2}-\mathrm{N}\right), 5.13\left(\mathrm{~d}, 2 \mathrm{H}, J=6.4 \mathrm{~Hz}, \mathrm{CH}_{2}-\mathrm{NH}\right), 6.91$ (brs, $\left.1 \mathrm{H}, \mathrm{NH}\right), 7.09-7.13$ (m, 2H, ArH), 7.29-7.39 (m, 5H, ArH), 7.71-7.75 (m, 2H, ArH).

Methyl carboxylate $(\mathbf{9 h})$

3-(butylamino)-2,5-dihydro-2-oxo-1-phenyl-1H-pyrrole-4-

Solid powder, mp: $60-62{ }^{\circ} \mathrm{C} ;{ }^{1} \mathrm{H}$ NMR $\left(400 \mathrm{MHz}, \mathrm{CDCl}_{3}\right): \delta 0.97(\mathrm{t}, 3 \mathrm{H}, J$ $\left.=7.2 \mathrm{~Hz}, \mathrm{CH}_{3}\right), 1.42\left(\right.$ sextet, $2 \mathrm{H}, J=7.2 \mathrm{~Hz}, \mathrm{CH}_{2}$ ), 1.64 (quintet, $2 \mathrm{H}, J=7.2$
$\left.\mathrm{Hz}, \mathrm{CH}_{2}\right), 3.82\left(\mathrm{~s}, 3 \mathrm{H}, \mathrm{OCH}_{3}\right), 3.85$ (t, $\left.2 \mathrm{H}, J=7.2 \mathrm{~Hz}, \mathrm{CH}_{-}-\mathrm{NH}\right), 4.45(\mathrm{~s}, 2 \mathrm{H}$, $\left.\mathrm{CH}_{2}-\mathrm{N}\right), 6.85(\mathrm{br} \mathrm{s}, 1 \mathrm{H}, \mathrm{NH}), 7.18(\mathrm{~d}, 1 \mathrm{H}, J=7.6 \mathrm{~Hz}, \mathrm{ArH}), 7.40(\mathrm{~d}, 2 \mathrm{H}, J=7.6$ $\mathrm{Hz}, \mathrm{ArH}), 7.73$ (d, 2H, $J=7.6 \mathrm{~Hz}, \mathrm{ArH})$.

Ethyl 1-phenyl-3-(phenylamino)-2,5-dihydro-2-oxo-1H-pyrrole-4carboxylate (9j)

Solid powder, mp: $139-141{ }^{\circ} \mathrm{C} ;{ }^{1} \mathrm{H}$ NMR $\left(400 \mathrm{MHz}, \mathrm{CDCl}_{3}\right): \delta 1.24(3 \mathrm{H}, \mathrm{t}$, $\left.J=7.2 \mathrm{~Hz}, \mathrm{OCH}_{\mathrm{CH}}\right), 4.24\left(2 \mathrm{H}, \mathrm{q}, J=7.2 \mathrm{~Hz}, \mathrm{OCH} \mathrm{CH}_{3}\right), 4.44(2 \mathrm{H}, \mathrm{s}, \mathrm{CH}-\mathrm{N})$, 7.15-7.23 (4H, m, ArH), 7.35 (2H, d, $J=7.6 \mathrm{~Hz}, \mathrm{ArH}), 7.41(2 \mathrm{H}, \mathrm{d}, J=7.6 \mathrm{~Hz}$, $\mathrm{ArH}), 7.82(2 \mathrm{H}, \mathrm{d}, J=7.8 \mathrm{~Hz}, \mathrm{ArH}), 8.01(1 \mathrm{H}, \mathrm{s}, \mathrm{NH})$.

Ethyl3-(4-bromophenylamino)-1-(4-bromophenyl)-2,5-dihydro-2-oxo-1Hpyrrole-4-carboxylate $(\mathbf{9 k})$

Solid powder, mp: $170-172{ }^{\circ} \mathrm{C} ;{ }^{1} \mathrm{H}$ NMR $\left(400 \mathrm{MHz}, \mathrm{CDCl}_{3}\right): \delta 1.24(3 \mathrm{H}, \mathrm{t}$, $\left.J=7.0 \mathrm{~Hz}, \mathrm{OCH}_{2} \mathrm{CH}_{3}\right), 4.24\left(2 \mathrm{H}, \mathrm{q}, J=7.2 \mathrm{~Hz}, \mathrm{OCH}_{2} \mathrm{CH}_{3}\right), 4.49\left(2 \mathrm{H}, \mathrm{s}, \underline{\mathrm{CH}}_{2}-\mathrm{N}\right)$, 7.09 (2H, d, $J=8.0 \mathrm{~Hz}, \mathrm{ArH}), 7.27-7.75(6 \mathrm{H}, \mathrm{m}, \mathrm{ArH}), 8.04(1 \mathrm{H}, \mathrm{s}, \mathrm{NH})$. Ethyl 4-(4-methylphenylamino)-1-(4-methylphenyl)-2, 5-dihydro-5-oxo-

1H-pyrrole-3-carboxylate $(\mathbf{9 m})$

Solid powder, mp:131-133 ${ }^{\circ} \mathrm{C} ;{ }^{1} \mathrm{H}$ NMR $\left(400 \mathrm{MHz}, \mathrm{CDCl}_{3}\right): 1.25(3 \mathrm{H}, \mathrm{t}$, $\left.J=7.2 \mathrm{~Hz}, \mathrm{CH}_{2} \mathrm{CH}_{3}\right), 2.37\left(6 \mathrm{H}, \mathrm{s}, 2 \mathrm{CH}_{3}\right), 4.23\left(2 \mathrm{H}, \mathrm{q}, J=7.2 \mathrm{~Hz}, 2 \mathrm{CH}_{2} \mathrm{CH}_{3}\right), 4.53$ $\left(2 \mathrm{H}, \mathrm{s}, \mathrm{CH}_{2}-\mathrm{N}\right), 7.06(2 \mathrm{H}, \mathrm{d}, J=8.4 \mathrm{~Hz}, \mathrm{ArH}), 7.14(2 \mathrm{H}, \mathrm{d}, J=8.4 \mathrm{~Hz}, \mathrm{ArH}), 7.21$ $(2 \mathrm{H}, \mathrm{d}, J=8.4 \mathrm{~Hz}, \mathrm{ArH}), 8.00(1 \mathrm{H}, \mathrm{s}, \mathrm{NH})$.

Ethyl 4-(4-methoxyphenylamino)-1-(4-methoxyphenyl)-2, 5-dihydro-5oxo-1H-pyrrole-3-carboxylate $(\mathbf{9 n})$

Solid powder, mp:153-155 ${ }^{\circ} \mathrm{C}$; ${ }^{1} \mathrm{H}$ NMR (400 $\left.\mathrm{MHz}, \mathrm{CDCl}_{3}\right): 1.26(3 \mathrm{H}$, $\left.\mathrm{t}, J=7.2 \mathrm{~Hz}, \mathrm{CH}_{2} \mathrm{CH}_{3}\right), 3.83\left(6 \mathrm{H}, \mathrm{s}, 2 \mathrm{OCH}_{3}\right), 4.23\left(2 \mathrm{H}, \mathrm{q}, J=7.2 \mathrm{~Hz}, \mathrm{CH}_{2} \mathrm{CH}_{3}\right)$, $4.50\left(2 \mathrm{H}, \mathrm{s}, \mathrm{CH}_{2}-\mathrm{N}\right), 6.87(2 \mathrm{H}, \mathrm{d}, J=8.8 \mathrm{~Hz}, \mathrm{ArH}), 6.93(2 \mathrm{H}, \mathrm{d}, J=8.8 \mathrm{~Hz}, \mathrm{ArH})$, $7.12(2 \mathrm{H}, \mathrm{d}, J=8.8 \mathrm{~Hz}, \mathrm{ArH}), 7.69(2 \mathrm{H}, \mathrm{d}, J=8.8 \mathrm{~Hz}, \mathrm{ArH}), 8.02(1 \mathrm{H}, \mathrm{s}, \mathrm{NH})$ Ethyl 3-(benzylamino)-1-phenyl-2,5-dihydro-2-oxo-1H-pyrrole-4carboxylate (9o)

Solid powder, mp:131-133 ${ }^{\circ} \mathrm{C}$; ${ }^{1} \mathrm{H}$ NMR $\left(400 \mathrm{MHz}, \mathrm{CDCl}_{3}\right): \delta 1.29(\mathrm{t}$, $\left.3 \mathrm{H}, J=7.2 \mathrm{~Hz}, \mathrm{OCH}_{2} \mathrm{CH}_{2}\right), 4.24\left(\mathrm{q}, 2 \mathrm{H}, J=7.2 \mathrm{~Hz}, \mathrm{OCH}_{2} \mathrm{CH}_{3}\right), 4.44(\mathrm{~s}, 2 \mathrm{H}$, $\left.\underline{\mathrm{CH}}_{2}-\mathrm{N}\right), 5.11\left(\mathrm{~d}, 2 \mathrm{H}, J=6.4 \mathrm{~Hz}, \underline{\mathrm{CH}}_{2}-\mathrm{NH}\right), 6.92(\mathrm{br}, 1 \mathrm{H}, \mathrm{NH}), 7.19-7.38(\mathrm{~m}, 8 \mathrm{H}$, $\mathrm{ArH}), 7.73-7.75$ (m, 2H, ArH).

Ethyl 1-(4-bromophenyl)-3-(butylamino)-2,5-dihydro-2-oxo-1H-pyrrole4-carboxylate ( $9 \mathrm{p})$

Solid powder, mp:93-95 ${ }^{\circ} \mathrm{C}$; IR $\left(\mathrm{KBr}, \mathrm{cm}^{-1}\right): v 3320(\mathrm{NH}), 1699(\mathrm{C}=\mathrm{O})$, $1649(\mathrm{C}=\mathrm{O}) ;{ }^{1} \mathrm{H}$ NMR $\left(400 \mathrm{MHz}, \mathrm{CDCl}_{3}\right): \delta 0.97$ (t, $\left.3 \mathrm{H}, J=7.2 \mathrm{~Hz}, \mathrm{CH}_{3}\right), 1.35$ (t, $3 \mathrm{H}, J=7.2 \mathrm{~Hz}, \mathrm{OCH}_{2} \mathrm{CH}_{3}$ ), 1.43 (sextet, $2 \mathrm{H}, J=7.6 \mathrm{~Hz}, \mathrm{CH}_{2}$ ), 1.61 (quintet, $\left.2 \mathrm{H}, J=7.6 \mathrm{~Hz}, \mathrm{CH}_{2}\right), 3.87(\mathrm{t}, 2 \mathrm{H}, J=7.2 \mathrm{~Hz}, \mathrm{CH}-\mathrm{NH}), 4.28(\mathrm{t}, 2 \mathrm{H}, J=7.2 \mathrm{~Hz}$, $\left.\mathrm{OCH}_{2} \mathrm{CH}_{3}\right), 6.72(\mathrm{br} \mathrm{s}, 1 \mathrm{H}, \mathrm{NH}), 4.40\left(\mathrm{~s}, 2 \mathrm{H}, \underline{\mathrm{CH}}_{2}^{2}-\mathrm{N}\right), 7.52(\mathrm{~d}, 2 \mathrm{H}, J=8.8 \mathrm{~Hz}$, $\mathrm{ArH}), 7.71(\mathrm{~d}, 2 \mathrm{H}, J=8.8 \mathrm{~Hz}, \mathrm{ArH})$

\section{CONCLUSION}

In summary, we have reported phthalic acid as a biodegradable green, economical and efficient catalyst for a simple and environmentally benign nature methodology to diverse synthesis of 3,4-dihydropyrimidin-2-(1H)-ones derivatives via one-pot three-component condensation Biginelli reaction of aldehyde derivatives $(1.0 \mathrm{mmol})$, urea/thiourea $(1.5 \mathrm{mmol})$ and ethyl $/ \mathrm{methyl}$ acetoacetate $(1.0 \mathrm{mmol})$ under thermal and solvent-free conditions and also, for the one-pot four-component synthesis of substituted dihydro-2-oxypyrrole by using of reaction of dialkyl acetylenedicarboxylate, formaldehyde and amines (aromatic and aliphatic). And phthalic acid as a biodegradable green, efficient and economical catalyst which has developed a new procedure in the field of green synthesis which has important benefits such as green, mild, biodegradable, non-toxic, high efficiently, environmentally benign nature, high catalytic activity, low-cost, eco-friendly.

\section{ACKNOWLEDGEMENTS}

We gratefully acknowledge financial support from the Research council of the University of Sistan and Baluchestan.

\section{REFERENCES}

1.- M.D. Nikam, P. Mahajan, A.V. Chate, S. Dabhade, C. Gill, J. Chil. Chem Soc. 60, 2847, (2015)

2.- M. Nasr-esfahani, M. Montazerohori, M. Aghel-mirrezaee, H.Kashi, J. Chil. Chem. Soc. 2014, 59, 2311, (2014)

3.- P.S. Anikt, N. Jaiprakash, N.B. Sangshetti, A.P. Dharbale, P.S. Sarkate wakte, D.B. Shinde, J. Chil. Chem. Soc. 2013, 58, 2200, (2013)

4.- Z. Madanifar, M.T. Maghsoodlou, M. Kangani, N. Hazeri, Res. Chem. Intermed. DOI: 10.1007/s11164-015-1993-6 (2015)

5.- P. Biginelli Gazz Chim Ital. 23, 360, (1893) 
6. - S. Wisen, J. Androsavich, C.G. Evans, L. Chang, J.E. Gestwicki , Bioorg Med. Chem. Lett.18, 60, (2008)

7.- O. Prakash, R. Kumar, V. Parkash, Eur. J. Med. Chem. 43, 435, (2008)

8.- A.D. Borthwick, A.J. Crame, P.F. Ertl, A.M. Exall, T.M. Haley, G.J. Hart, A.M. Mason, M.K. Pennell, O.M.P. Singh, G.G. Weingarten, J.M. Weingarten, J. Med. Chem. 45, 1, (2002)

9.- R. Shiraki, A. Sumino, K. Tadano, S. Ogawa, Tetrahedron Lett. 36, 5551, (1995)

10.- Y. Chen, D.X. Zeng, N. Xie, Y.Z. Dang, J. Org. Chem. 70, 5001, (2005)

11.- B.B. Snider, B.J. Neubert, J. Org. Chem. 69, 8952, (2004)

12.- I.A. Khodja, R. Boulcina, A. Debache, J. Chem. Pharm. Res. 6,1040, (2014)

13.- Y. Zhang, B. Wang, X. Zhang, J. Huang, C. Liu, Molecules. 20, 3811, (2015)

14.- S.B. Singh, M.A. Goetz, E.T. Jones, G.F. Billes, R.A. Giacobbe, L. Herranz, S. Stevens Miles, D.L. Williams, J. Org. Chem. 60, 7040, (1995)

15.- S. Chitra, K. Pandiarajan, TetrahdronLett. 50, 2222, (2009)

16.- A. Kamal, T. Krishnaji, M.A. Azhar, J.Catal. Commun. 8, 1929, (2007)

17.- J. Lai, M. Sharma, S. Gupta, P. Parashar, P. Sahu, D.D. Agarwal, J. Mol. Catal. A. Chem. 352, 31, (2012)

18.- A. Kumar, R.A. Maurya, Tetrahedron Lett. 48, 4569, (2007)
19.- M. Litvic, I. Vecenaj, Z.M. Ladisic, M. Lovric, V. Vinkovic, M. FilipanLitvic, Tetrahedron. 66, 3463, (2010)

20.- C.J. Liu, J.D. Wang, Molecules. 14, 763, (2009)

21.- A.T. Khan, A. Ghosh, M. Musawwer Khan, Tetrahedron Lett. 53, 2622, (2012)

22.- Q. Zhu, H. Jiang, J. Li, S. Liu, C. Xia, M. Zhang, J. Comb. Chem. 11, 685, (2009)

23.- S. S. Sajadikhah, N. Hazeri, J. Res. Chem. Intermed. 40, 737, (2014)

24.- S.S. Sajadikhah, N. Hazeri, M.T, Maghsoodlou, S.M. Habibi Khorasani, A. Beigbabaei, A. C. Willis., J. Iran. Chem. Soc. 10, 863, (2013)

25.- S.S. Sajadikhah, N. Hazeri, M.T. Maghsoodlou, J. Chem. Res. 37, 40, (2013)

26.- S.S. Sajadikhah, M.T. Maghsoodlou, N. Hazeri, S. Mohamadian-Souri, Res. Chem. Intermed. DOI: 10.1007/s11164-015-2178-z (2015)

27.- S.S. Sajadikhah, M.T. Maghsoodlou, N. Hazeri, Chin. Chem. Lett. 25, 58, (2014)

28.- S. Sajjadifar, M.A. Zolfi gol, A. Ghorbani-Choghamarani, A.R. Banaei, S Karimian, S. Miri Scientia. Iranica. 21, 2005, (2014)

29.- S. Sajjadifar, S. Miri, Int. J. Chem. Tech. Res. CODEN (USA): IJCRGG. $6,5433(2014)$

30.- F.M. Danikuu, E.N.K. Sowley, J. Med \& Biomed. Sci. 3, 9, (2014) 